\title{
DESARROLLO DE CULTIVARES DE PAPA RESISTENTES A LA PALOMILLA Phthorimaea operculella (Zeller), EN COLOMBIA
}

\author{
NELSON ESTRADA R., LUIS VALENCIA V.*
}

\section{RESUMEN}

Se utilizaron varias especies de papa, silvestres y cultivadas, para obtener híbridos que se probaron para su resistencia a la "palomilla" en el laboratorio. Varios de ellos mostraron una alta resistencia a la "palomilla" en el tubérculo lo cual se supone que sea debido a la rápida formación de sustancias tóxicas cuando el tubérculo es herido por las larvas del insecto. Se informa sobre un clone muy promisorio obtenido con alta resistencia al insecto que además es muy productivo, de buena calidad culinaria y que tiene tolerancia al "tizón tardío" causado por Phytophthora infestans.

Palabras Claves Adicionales: tricomas, polifenoles, melanina, sólidos totales, polen 2n, Phytophthora infestans.

\section{ABSTRACT}

Development of potato cultivars resistant of tuber moth, Pthorimaea operculella (Zeller), in Colombia.

Several wild and cultivated Potato species were utilized to obtain hybrids which were tested for tuber moth resistance in the laboratory. Some of these new hybrids showed a high resistance to tuber moth in the tubers. It is postulated that this resistance is caused by the action of toxic substances generated when the tuber moth larvae start eating the tubers. It was found a promising done that had a high resistance to the insect, in adition to high yielding, good cooking quality and tolerance to late blight caused by Phytophthora infestans.

Additional Index Words: trichomes, polyphenols, melanine, total solids, 2n pollen, Phytophthora infestans.

La "palomilla" de la papa, Phthorimaea operculella, es un insecto que causa serios daños en el follaje y especialmente en los tubérculos de papa, pudiéndose considerar como una plaga limitante del cultivo que se halla presente en Colombia y en todos los países tropicales del mundo que cultivan papa.

Profesor asociado, Facultad de Agronomía, Universidad Nacional y fitomejorador, ICA. Apartado Aéreo 151123 Bogotá. Entomólogo Región I Zona Andina, Centro Internacional de la Papa, CIP, Bogotá. 
Su daño en el follaje se caracteriza por galerías que produce la larva en las hojas y tallos lo cual reduce el área fotosintética y la eficiencia de la planta. Además, el daño más grave lo produce en los tubérculos ya que el insecto adulto deposita los huevos en los ojos de los tubérculos y al eclosionar los huevos las larvas inician la perforación y formación de túneles en el interior del mismo, contaminándolo con excrementos y haciendo daños graves que lo inutilizan para el consumo.

Estos daños se pueden producir en el campo, antes de la cosecha, sobre todo en los tubérculos más cercanos a la superficie, porque la hembra del insecto adulto se introduce por muy pequeñas hendiduras que el suelo puede presentar, y también durante el almacenamiento a granel, en empaques en la bodega, o durante el transporte.

El control con plaguicidas resulta muy difícil por los hábitos del insecto, porque afecta además, a los tubérculos que son el producto comestible de la planta y porque los plaguicidas originan problemas graves como toxicidad para el hombre por su efecto residual en el producto aún después de un lapso de tiempo de aplicación, y por los problemas bien conocidos de contaminación ambiental que perjudica al hombre y los animales útiles.

La presente investigación se ha desarrollado en Colombia considerando que se ha detectado resistencia genética en especies silvestres y aún en híbridos especiales efectuados entre especies cultivadas constituyendo así una alternativa más económica y saludable para el control de esta plaga.

Se han utilizado especies silvestres y cultivadas en cruzamientos que resulta largo enumerar aquí, (Estrada y Valencia (1987), Scurrah y Raman (1984). Solo se reportan ahora algunas familias y clones valiosos. Los resultados han sido muy alentadores y abren la posibilidad de seleccionar híbridos y nuevas variedades de papa con alta resistencia a otras plagas muy graves y económicas como el "gusano blanco" o "taladrador del tubérculo" (Premnotripes vorax) y el "gusano minador del tubérculo" (Liriomyza ecuadoriensis). La explicación y conclusión para obtener resistencia genética en estos nuevos clones de papa es que se trata de un tipo de resistencia bioquímico, hasta cierto punto inmunológico que promueve la producción de ciertos compuestos antibióticos - polifenoles y otros- que actúan contra las larvas que tratan de penetrar en el tubérculo cuando le causan a éste ligerísimas heridas.

\section{MATERIALES Y MÉTODOS}

Esta investigación se desarrolló en los invernaderos y campos del Centro Nacional de Investigaciones "Tibaitatá", en el laboratorio de Entomología del Centro Internacional de la Papa (CIP), ubicado en la misma sede y en el Centro de Investigación Regional San Jorge, del 
ICA. Todas las pruebas de resistencia a la palomilla fueron con tubérculos.

Los materiales o clones de papa que se probaron incluyeron:

a) Especies silvestres como Solanum sparsipilum, S. berthaultii, $S$. polyadenium, $S$. chacoense y otras enviadas por el proyecto IR-1 del Departamento de Agricultura de los Estados Unidos (U.S.D.A.), localizado en Sturgeon Bay, Wisconsin. S. berthaultii es notable por su gran número y calidad de tricomas o pelos glandulares que posee en el follaje y que inhiben los ataques del insecto en el follaje.

b) Híbridos procedentes de los programas de mejoramiento genético del Centro Internacional de la Papa (CIP) con sede en Lima, Perú.

c) Clones de las especies cultivadas de papa Solanum andigena y Solanum phureja de la Colección Central Colombiana de papas mantenida por el Instituto Colombiano Agropecuario (ICA).

d) Híbridos que fueron obtenidos en el Centro Nacional de Investigaciones "Tibaitatá" en el desarrollo de esta investigación.

Los resultados de las pruebas de resistencia se obtuvieron en el laboratorio y fueron conducidas por el autor de este artículo.

El laboratorio estuvo acondicionado con temperatura constante $26^{\circ} \pm 2$ C) que favorecía la reproducción del insecto y humedad relativa ambiental de 50\%-80\%, Para las pruebas de resistencia que se mencionan en este trabajo la "parcela experimental" consistió de dos tubérculos de aproximadamente $70 \mathrm{~g}$. cada uno de ellos, colocados en un envase plástico que tenía un piso o cama de arena lavada de un centímetro de espesor. Cada tubérculo fue infestado artificialmente colocándose 10 larvas de primer estadio y cada clone de papa tuvo tres repeticiones. A los 20 días de iniciada la prueba se contó el número de pupas por parcela, examinando y cortando los tubérculos. Los daños fueron transformados a la raíz cuadrada para efectuar el análisis estadístico. En varios casos, los experimentos fueron repetidos para reconfirmar los resultados. Estas investigaciones se han venido desarrollando por cinco años desde 1982 hasta 1987.

\section{RESULTADOS Y DISCUSIÓN}

Las Tablas 1 y 2 muestran varias familias y clones evaluados, parte procedente del CIP y parte obtenidas por los autores en Colombia. De estos últimos híbridos merece mencionarse especialmente el clone obtenido en 1982 y que tiene el No. 82-3-5. Este clone ha mostrado muy alta resistencia en mas de cuatro pruebas de laboratorio, pruebas en silos 
nísticos y en varias observaciones de campo. Tiene como atributos, fuera de su alta resistencia a la palomilla, calidad culinaria de tubérculo muy buena y resistencia a la "gota" causada por el hongo Phythophthora infestans.

TABLA 1. Pruebas de clones para resistencia a la palomilla en Colombia.

\begin{tabular}{|c|c|c|c|c|}
\hline \multirow{2}{*}{ Clone o familia } & \multirow{2}{*}{\multicolumn{2}{|c|}{ Genealogía }} & \multicolumn{2}{|c|}{ Clones } \\
\hline & & & evaluados & resistentes \\
\hline Och 6759 & \multicolumn{2}{|l|}{ S. andigena } & 20 & 14 \\
\hline $82-3$ & \multicolumn{2}{|l|}{ S. tuberosum x S. phureja } & 5 & 1 \\
\hline LV-1 & P-83755 tbr x (spl x tbr) & (spl x tbr) & 1 & 1 \\
\hline LV-3 & P-83758 & “ & 4 & 4 \\
\hline LV-4 & \multicolumn{2}{|l|}{ F-743-4 tbr x (spl x tbr) } & 3 & 3 \\
\hline LV-7 & T A-3 “ & & 1 & 1 \\
\hline LV-13 & E-656-1 “ & & 3 & 3 \\
\hline \multirow[t]{2}{*}{ LV-20 } & P-83630 tbr x (spl x tbr) & x (spl x tbr) & 1 & 1 \\
\hline & & Total & 38 & 28 \\
\hline
\end{tabular}

TABLA 2. Promedio de pupas por parcela, obtenidas de una prueba experimental para resistencia a la palomilla, empleando material resistente proveniente del CIP.

\begin{tabular}{|c|c|c|c|}
\hline Clone & Genealogía & $\begin{array}{c}\text { Promedio } \\
\text { Pupas } \\
*\end{array}$ & Resistencia \\
\hline P-83755 & tbr (spl x tbr) $\times \quad(\operatorname{spl} x$ tbr $)$ & $1.67 \mathrm{a}$ & AR \\
\hline TA-3 & tbr x (spl x tbr) & 2.67 a & AR \\
\hline E-656-1 & " " " & $3.00 \mathrm{ab}$ & AR \\
\hline P-83758.1 & tbr x (spl x tbr) x (spl x tbr) & 3.67 abe & AR \\
\hline Parda pastusa & S. andigena & 3.67 abe & AR \\
\hline E-656-1 x masal & tbr x (spl x tbr) & 4.33 abe & $\mathrm{AR}$ \\
\hline F-743-4 x masal & " " " " & 5,00 abe & AR \\
\hline P-83758.2 & tbr $\times($ spl $\times$ tbr $) \times($ spl $\times$ tbr $)$ & 6.00 abcd & AR \\
\hline P-83758.3 & " " " & 8.33 bcd & $\mathrm{R}$ \\
\hline F-743-4 x masal & tbr x (spl x tbr) & $11.00 \mathrm{~d}$ & $\mathrm{R}$ \\
\hline S. phureja (CСC 81) & S. phureja & 19.00 & AS \\
\hline
\end{tabular}

* Letras diferentes indican alta significación $(\mathrm{P}=0.01)$ para la prueba de Duncan.

AR Alta resistencia

$\mathrm{R}$ Resistencia

AS Alta

susceptibilidad 
La Tabla 3 ilustra el comportamiento del clone 82-3-5 en el experimento llevado a cabo en 1987 en el Centro de Investigación Regional San Jorge, donde mostró rendimientos superiores a los conocidos cultivares ICA-Puracé, ICA-Guantiva, Diacol, Capiro, Diacol Monserrate y Parda Pastusa. Este clone se seleccionó después de cinco años de observaciones dentro de la familia 82-3 que se componía de otros 13 clones. Fue obtenido mediante el cruzamiento del cultivar Atzimba de México, que pertenece a la especie tetraploide Solanum tuberosum y el cultivar diploide "Yema de huevo" de la especie Solanum phureja y que se caracteriza por su excelente calidad y precocidad. El clone 82-3-5 resultante de esta hibridación es tetraploide debido a la utilización de gametos 2n (no reducidos) en el polen del cultivar Yema de Huevo, empleando aquí la técnica descrita por Mendiburu y Peloquin (1971) para transferir genéticamente en forma intacta los buenos caracteres de calidad del cultivar Yema de Huevo.

TABLA 3. Producción en el CRI San Jorge de 8 clones de papa durante la siembra de año grande, 1987. El híbrido 82-3-5 altamente resistente a la palomilla muestra el buen rendimiento.

\begin{tabular}{lccc}
\hline CLONE & $\begin{array}{c}\text { Rendimiento } \\
\text { Kg /parcela }\end{array}$ & $\begin{array}{c}\text { Rendimiento } \\
\text { equiv. t /ha }\end{array}$ & $\begin{array}{c}\text { Significancia } \\
1 \% 6 \mathrm{Kg} / \text { promedio }\end{array}$ \\
\hline 82-3-5 & 93.0 & 39.0 & $\mathrm{a}$ \\
Puracé & 84.0 & 35.0 & $\mathrm{~b}$ \\
$79-94-3$ & 75.0 & 31.0 & $\mathrm{c}$ \\
Capiro & 67.0 & 28.0 & $\mathrm{~d}$ \\
Monserrate & 58.0 & 24.0 & $\mathrm{e}$ \\
Guantiva & 55.0 & 23.0 & $\mathrm{e}$ \\
Pastusa & 51.0 & 21.0 & $\mathrm{f}$ \\
380073-2 & 42.0 & 18.0 & $\mathrm{~g}$ \\
\hline
\end{tabular}

Las diferencias entre letras diferentes son significativas al $1 \%$.

En la figura 1 se muestra un clone híbrido de $S$. sparsipilum, $S$. phureja CCC 81.

En la figura 2 se puede observar la alta resistencia del clone 823-5 en comparación con cultivares susceptibles en pruebas controladas de laboratorio.

En la figura 3 se nota el desarrollo vigoroso y uniforme del crecimiento del follaje del clone 82-3-5 en el campo, Centro Regional de Investigación San Jorge.

En la figura 4 se ve el buen tipo de tubérculo que produce, que son casi redondos, isodiamétricos, piel de color rosado y ojos superficiales.

En la figura 5 se observa la producción del clone 82-3-5 al cosechar la parcela. 


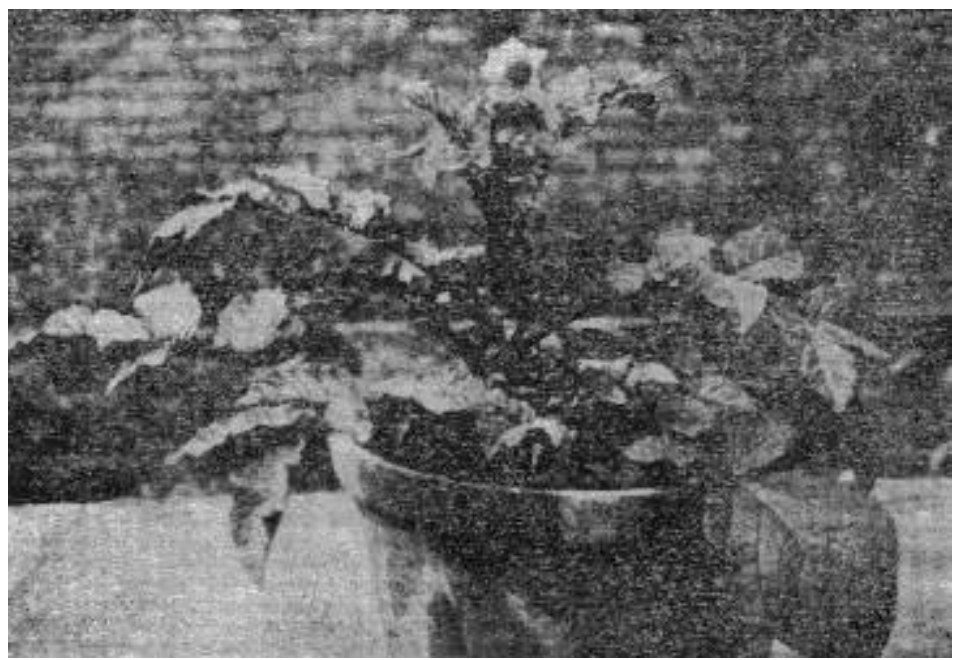

FIGURA 1. Híbrido obtenido del cruzamiento de Solanum sparsipilum resistente a la palomilla y Solanum phureja cultivar Yema de Huevo CCC 81.

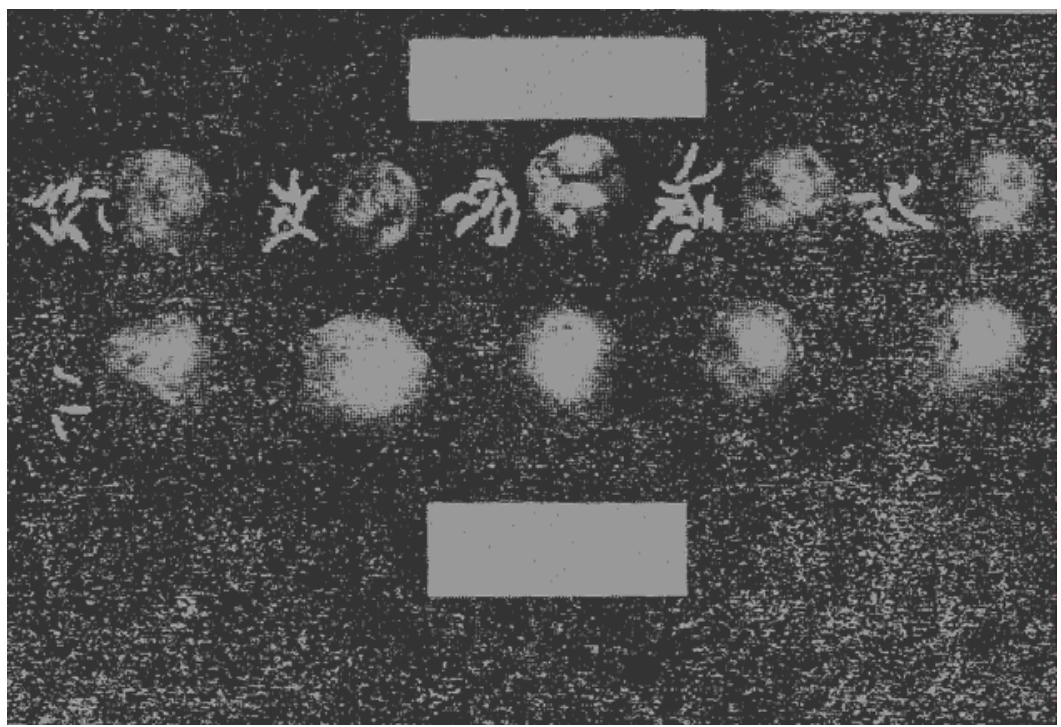

FIGURA 2. Resultado de una prueba de laboratorio con larvas de palomilla (Phthorimaea operculella). Arriba, cinco tubérculos del cultivar diploide $(2 n=24)$ Yema de Huevo CCC 81. Abajo, cinco tubérculos del híbrido 82-3-5. 


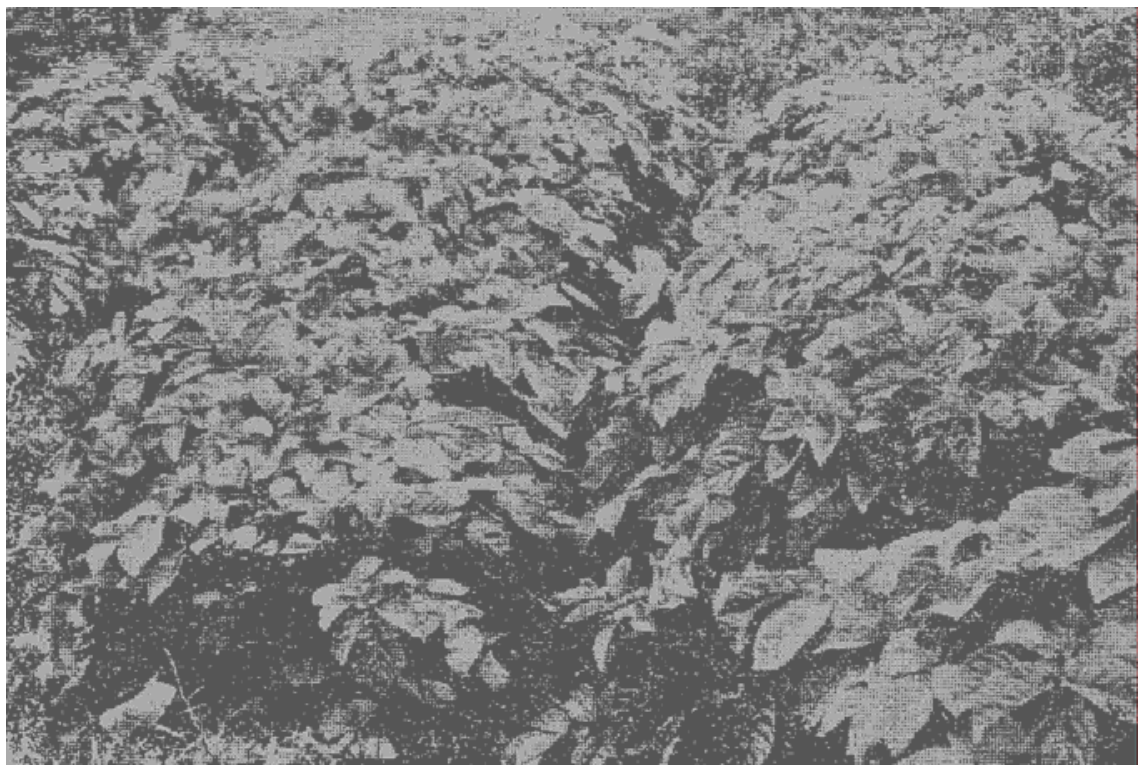

FIGURA 3. Desarrollo vigoroso y sano del híbrido 82-3-5, creciendo en el Centro Regional de Investigaciones San Jorge, mostrando un tipo de follaje y arquitectura de planta muy eficiente.

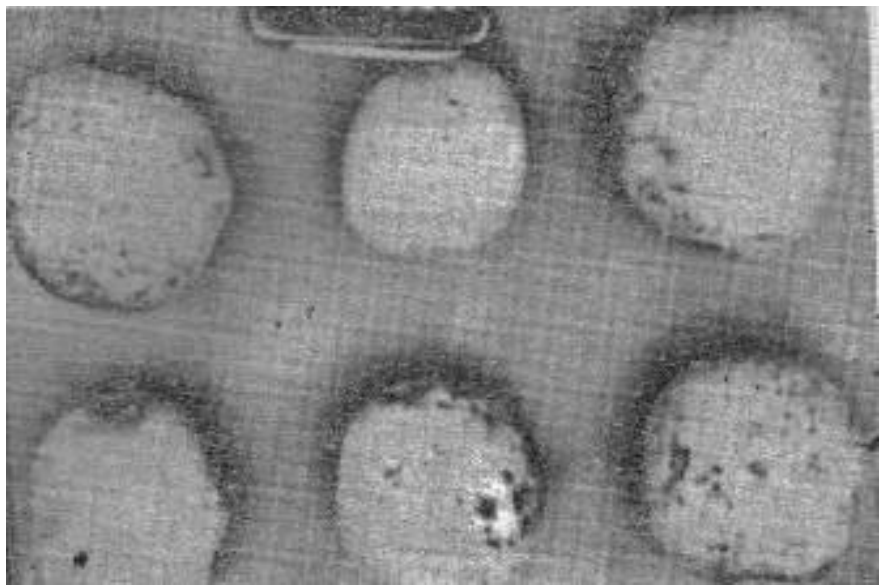

FIGURA 4. Tubérculo del híbrido 82-3-5 que ilustran su buena forma y tamaño y ojos superficiales. 


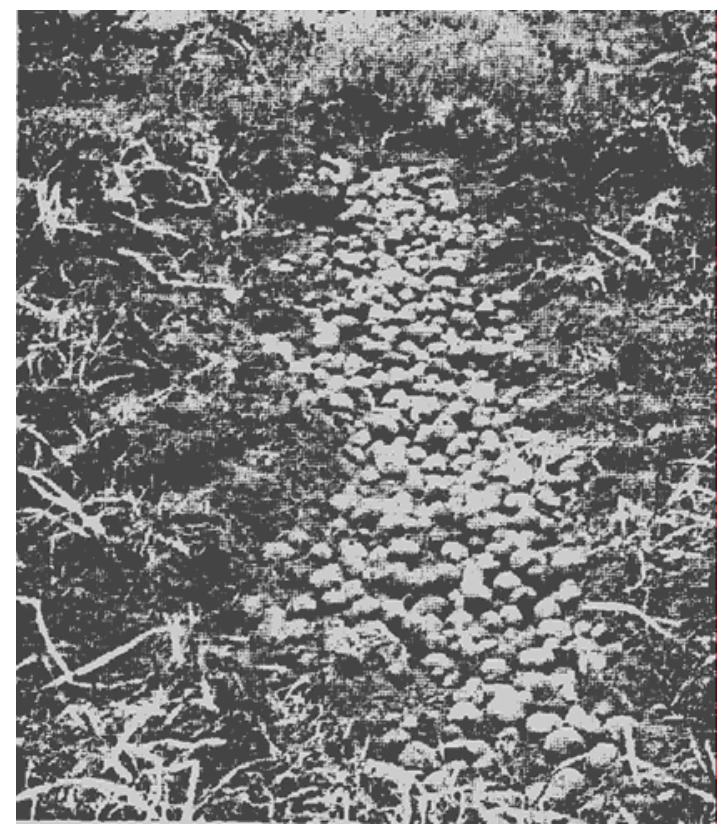

FIGURA 5. Cosecha del híbrido 82-3-5 en el CRI San Jorge, donde mostró su buena adaptación y capacidad productiva que compitió con los mejores cultivares.

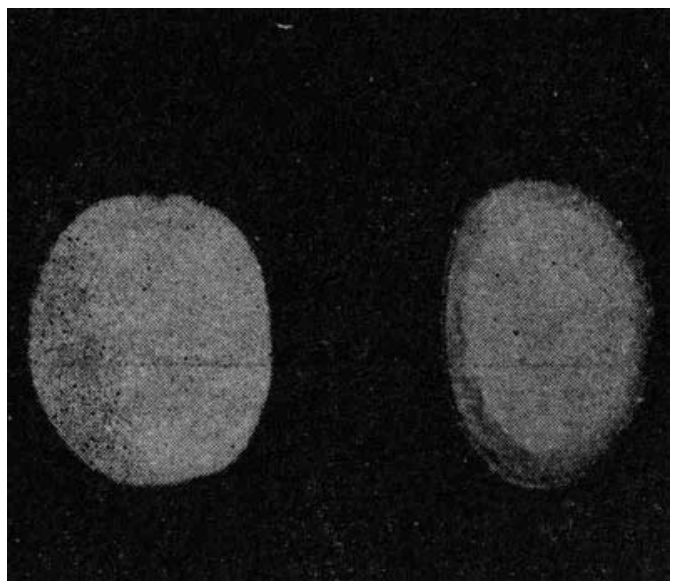

FIGURA 6. Clone 82-3-5. Obsérvese el tejido oscuro de melanina inmediatamente debajo de la piel del tubérculo a la derecha. Esta es la barrera que probablemente protege al tubérculo del daño de larvas de "palomilla". 
En la figura 6 se observa claramente la reacción del tubérculo cuando recibe heridas de agentes extraños, y que hace producir el antibiótico, probable causa de su alta resistencia a la palomilla. Esta sustancia se inactiva y descompone con la cocción y por ello los tubérculos no se afectan para el consumo humano en su apariencia o nutrición.

Finalmente la Tabla 4 muestra datos de evaluación del contenido de sólidos totales, peso específico y calidad culinaria. Por los datos anteriores puede decirse que el clone 82-3-5 tiene las características necesarias para ser entregada a los cultivadores de papa como nuevo cultivar con resistencia a la palomilla, a la "gota" y con buena precocidad y alta producción.

TABLA 4. Datos comparativos de peso específico y calidad, de clones resistentes a la palomilla y algunos cultivares comunes.

\begin{tabular}{lccc}
\hline Variedad o clone & Peso específico & $\begin{array}{c}\text { Solidos totales } \\
\%\end{array}$ & $\begin{array}{c}\text { Calidad } \\
\text { culinaria }\end{array}$ \\
\hline ICA Puracé & 1.080 & 19.8 & buena \\
1CA Guantiva & 1.085 & 20.6 & buena \\
Diacol Capiro & 1.087 & 21.0 & buena \\
Diacol Monserrate & 1.095 & 22.6 & muy buena \\
Parda Pastusa & 1.090 & 21.6 & muy buena \\
79-94-3 & 1.086 & 20.8 & buena \\
82-3-5 & 1.085 & 20.6 & muy buena \\
\hline
\end{tabular}

\section{Agradecimientos}

- Instituto Colombiano Agropecuario, ICA, Colombia

— Centro Internacional de la Papa, CIP, Perú

- Inter-Regional Potato Introduction Project IR-1, USDA y University of Wisconsin, USA. 


\section{REFERENCIAS BIBLIOGRÁFICAS}

1. Estrada, N.; Valencia, L. 1987. Mejoramiento de la Papa para resistencia a la palomilla (Phthorimaea operculella (Zeller) ) en Colombia. Asociación Latinoamericana de la Papa, Memorias, p. 212-219.

2. Mendiburu, A. O.; Peloquin, S. J. 1971. High yield tetraploids from 4x - 2x and 2x-2x matings. Am. Potato J. 48: 300-801.

3. Raman, K. V. Palacios, M. 1982. Screening potato for resistance to Tuber moth (Phthorimaea operculella) in Solanum tuberosum. J. Econ. Entomol 75: 47-49.

4. Scurrah, M.; Raman, K. V. 1984. Breeding and screening to major potato pests. CIP, XXII Planning Confer. Integrated Pest Management, Lima, Perú. 